\title{
Faster Model Checking for the Modal Mu-Calculus
}

\author{
Rance Cleaveland* ${ }^{*} \quad$ Marion Klein ${ }^{\dagger} \quad$ Bernhard Steffen $^{\dagger}$
}

\begin{abstract}
In this paper, we develop an algorithm for model checking that handles the full modal mucalculus including allernaling fixpoints. Our algorithm has a better worst-case complexity than the best known algorithm for this logic while performing just as well on eertain sublogies as other specialized algorithms. Important for the efficiency is an alternative characterisation of formulas in terms of equational systems, which enables the sharing and reuse of intermediate results.
\end{abstract}

\section{Introduction}

Much work in the field of automated verification has focused on finite-state transition systems (or automata) as models for system behavior [CES, CPS1, CPS2, Fe, MSGS, RRSV, RdS]. The modal mu-calculus [Ko] is a particularly useful logic for reasoning about such models; not only may a number of temporal logics for expressing system properties be translated into it [EL], but it may also be used to encode various behavioral equivalences and preorders [Ste, SI]. Thus, this logic supports algebraic as well as logic-based approaches to verification.

In this paper, we present an algorithm for determining when states in a finite-state transition system possess properties expressed in the modal mu-calculus. Our model-checking algorithm improves on the best existing methods for model checking in this logic [A, EL] while performing just as well on certain sublogics as specialized algorithms (cf. [CS1, CS2]). Important for the efficiency is an alternative characterization of formulas in terms of equational systems, which enables the sharing and reuse of intermediate results.

The remainder of the paper is organized as follows. In the next section we present the syntax and semantics of the mu-calculus, and in the section following we give an alternative, equation-based presentation of this logic. Section 4 presents our model-checking algorithm, while the subsequent section establishes its correctness and complexity. The paper closes with a detailed discussion of an example in Section 6 and some conclusions and directions for future work in Section 7.

\section{Syntax and Semantics of the Mu-Calculus}

This section first provides a brief overview of labeled transition systems, which are used as models for the mu-calculus. Then the syntax and semantics of the logic are developed.

\subsection{Transition Systems}

Definition $2.1 A$ labeled transition system $T$ is a triple $\langle\mathcal{S}$, Act, $\rightarrow\rangle$, where $S$ is a set of states, Act is a set of actions, and $\rightarrow \subseteq S \times A c t \times S$ is the transition relation.

Intuitively, a labeled transition system encodes the operational behavior of a system. The set $\mathcal{S}$ represents the set of states the system may enter, and Act contains the set of actions the system may perform. The relation $\rightarrow$ describes the actions available to states and the state transitions that

\footnotetext{
-Department of Computer Science, North Carolina State Univervity, Raleigh, NC 27695-8206, USA - The author was supported by NSF Grant CCR-9014775.

'Lehrstuhl fux Informatik II, RWTHI-Awchen, Ahornstrafle 55, W-5100 Aachen, GERMANY - Part of the work has been funded by DFG Grant Ste 537/2-1.
} 
Formulas are interpreted with respect to a fixed labeled transition system $\langle\mathcal{S}$, Act $\rightarrow\rangle$, a valuation $\mathcal{V}: \mathcal{A} \rightarrow 2^{\mathcal{S}}$, and an environment $e: \operatorname{Var} \rightarrow 2^{\mathcal{S}}$.

$$
\begin{aligned}
\llbracket A \rrbracket e & =\mathcal{V}(A) \\
\llbracket X \rrbracket e & =e(X) \\
\llbracket \neg \Phi] e & =S \backslash \llbracket \Phi] e \\
\llbracket \Phi_{1} \wedge \Phi_{2} \rrbracket e & =\llbracket \Phi_{1} \rrbracket e \cap \llbracket \Phi_{2} \rrbracket e \\
\llbracket[a] \Phi \rrbracket e & =\left\{s \mid \forall s^{\prime} . \Delta \stackrel{a}{\rightarrow} s^{\prime} \Rightarrow s^{\prime} \in[\Phi] e\right\} \\
\llbracket \nu X . \Phi] e & =\bigcup\left\{S^{\prime} \subseteq \mathcal{S} \mid S^{\prime} \subseteq[\Phi] e\left[X \mapsto S^{\prime}\right\rceil\right\}
\end{aligned}
$$

Figure 1: The Semantics of Formulas.

may result upon execution of the actions. In the remainder of the paper we use $s \stackrel{a}{\rightarrow} s^{\prime}$ in lieu of $\left\langle s, a, s^{\prime}\right\rangle \in \rightarrow$, and if $s \stackrel{a}{\rightarrow} s^{\prime}$ then we say that $s^{\prime}$ is an a-derivative of $s$. Finally, we refer to a labeled transition system as finile-stale when $\mathcal{S}$ and $A c t$ are finite.

\subsection{Syntax and Semantics of Formulas}

The syntax of the modal mu-calculus is parameterized with respect to a (countable) set Var of variables, a set $\mathcal{A}$ of atomic propositions, and a set $A c t$ of actions. For technical reasons we assume that $\mathcal{A}$ is closed with respect to negation: for every $A \in \mathcal{A}$ there is a $B \in \mathcal{A}$ that is semantically equivalent to the negation of $A$. In what follows, $X$ will range over $\operatorname{Var}, A$ over $\mathcal{A}$, and $a$ over $A c t$ The syntax of formulas may be given by the following grammar.

$$
\Phi::=A|X| \neg \Phi|\Phi \wedge \Phi|[a] \Phi \mid \nu X . \Phi
$$

The maximum fixpoint operator $\nu$ binds free occurrences of $X$ in $\Phi$ in the usual sense. We impose an additional syntactic restriction on formulas of the form $\nu X . \Phi$ : each free occurrence of $X$ in $\Phi$ must be within the scope of an even number of negations. This requirement ensures the well-definedness of the semantics of the logic.

Let $\Phi[X:=\Gamma]$ represent the formula obtained by simultaneously substituting the formula $\Gamma$ for the free occurrences of the variable $X$ in $\Phi$. Then we may also define the usual dual operators to the ones we have presented.

$$
\Phi_{1} \vee \Phi_{2}=\neg\left(\neg \Phi_{1} \wedge \neg \Phi_{2}\right) \quad\langle a\rangle \Phi=\neg[a](\neg \Phi) \quad \mu X . \Phi=\neg \nu X . \neg(\Phi[X:=\neg X])
$$

In what follows we say that $\Phi^{\prime}$ is a proper subformula of $\Phi$ if it is a subformula of $\Phi$ that is not $\Phi$ itself. Given a formula, its top-level subformulas with a certain property are defined to be those maximal proper subformulas having the property. A formula is said to be a $\nu$-formula ( $\mu$-formula) if it has the form $\nu X . \Phi(\mu X$. $\Phi)$ for some $X$ and $\Phi$. We refer to a formula as closed if it contains no free variables and simple if it is fixpoint-free and contains only variables and atomic propositions as proper subformulas. For example, $X_{1} \wedge A_{2}$ is simple, while $(a)\left(X_{3} \vee X_{1}\right)$ is not.

The formal semantics of formulas appears in Figure 1 . It is given with respect to a finite-state labeled transition system $\langle\mathcal{S}, A c h \rightarrow\}$, a valuation $\mathcal{V}$ mapping atomic propositions to subsets of $\mathcal{S}$, and an environment $e$ mapping variables to subsets of $\mathcal{S}$. Note that $e[X \mapsto S]$ is the environment that results by updating the binding of $X$ to $S$ in $e$.

Intuitively, the semantic function maps a formula to the set of states for which the formula is "true". Accordingly, a state $s$ satisfies $A \in \mathcal{A}$ if $s$ is in the valuation of $A$, while $s$ satisfies $X$ if $s$ is an element of the set bound to $X$ in $e$. The propositional constructs are interpreted in the usual fashion: $s$ satisfies $\neg \Phi$ if it does not satisfy $\Phi$ and satisfies $\Phi_{1} \wedge \Phi_{2}$ if it satisfies $\Phi_{1}$ as well as $\Phi_{2}$. The construct $[a]$ is a modal operator; $s$ satisfies $[a] \Phi$ if each $a$-derivative of satisfies $\Phi$. 
The syntactic restriction on the bodies of $\nu$-formulas and the semantics of the other logical connectives ensures that semantically, the bodies give rise to monotonic functions (on the lattice sets of states) [C]. Accordingly, on the basis of the Knaster-Tarski Fixpoint Theorem [T] the semantics of $\nu X . \Phi$ is given as the greatest fixpoint of the monotonic function corresponding to $\Phi$. In addition, for finite-state labeled transition systems the bodies of $\nu$-formulas are continuous, and Kleene's Fixpoint Theorem then provides the following iterative characterization of the semantics. Define $\phi_{i}$ by $\phi_{0}=\mathcal{S}$ and $\left.\phi_{i+1}=\llbracket \Phi\right] e\left[X \mapsto \phi_{i}\right]$ for $i \geq 1$. Then $[\nu X$. $\Phi] e=\bigcap_{i=0}^{\infty} \phi_{i}$. Formula $\mu X$. $\Phi$ can be characterized dually as $\bigcup_{i=0}^{\infty} \hat{\phi}_{i}$, where $\hat{\phi}_{0}=0$ and $\hat{\phi}_{i+1}=[\phi] e\left[X \mapsto \hat{\phi}_{i}\right]$. The next lemma establishes that the meaning of a closed formula does not depend on its environment.

Lemma 2.2 Fix a finite-state transition system and valuation, and let $\Phi$ be a closed formula. Then for any environments $e$ and $e^{\prime}$ we have: $\left[\Phi \rrbracket e=[\Phi] e^{\prime}\right.$.

The lemma holds because all variables in closed formulas are bound by a fixpoint operator, and this excludes any influence of the initial environment on the semantics of the formula. We therefore omit reference to an environment for closed formulas and write [ $\Phi]$. Finally, it is also possible to translate formulas into positive normal form (PNF), i.e. into a negation-free formula in which no variable is bound more than once. This is a consequence of the following lemma, where $|\Phi|$ represents the number of occurrences of operators and atomic formulas in $\mathbf{\Phi}$.

Lemma 2.3 Let $\Phi$ be a closed formula in the modal $\mu$-calculus. Then can be translated into a closed formula $\Phi^{\prime}$ in the logic extended with $V,\langle a\rangle$ and $\mu$ in $O(|\Phi|)$ time such that
1) $\Phi^{\prime}$ is negation-free,
2) $[\Phi \rrbracket=\llbracket \Phi \backslash$ and
3) $\left|\Phi^{\prime}\right| \leq|\Phi|$.

The translation is done by "driving" negations inside the subformulas in the standard way following DeMorgans Laws etc, and renaming variables as appropriate. The resulting formula $\Phi$ ' is not larger than $\Phi$ because of our assumptions that all free occurrences of variables in fixpoint formulas must be inside the range of an even number of negations and that the atomic propositions are closed under negation.

For notational simplicity, in what follows we only consider formulas whose top-level operator is a fixpoint operator. This is not a serious restriction, as the semantics of other formulas can be trivially determined in linear time once the semantics of the top-level fixpoint formulas have been computed.

\subsection{Alternation Depth of Formulas}

The complexity of the algorithm that we present in the following sections will depend on a measure on formulas called alternation depth. Intuitively, the alternation depth of a formula is the length of a maximal "chain" of mutually recursive greatest and least fixpoint subformulas (cf. [EL]).

Definition 2.4 (Alternation Depth of Formulas) Let be in PNF. Then the alternation depth, ad( $\Phi)$, of $\Phi$ is defined inductively as follows.

- If $\Phi$ contains closed top-level fixpoint-subformulas $\Gamma_{1}, \ldots, \Gamma_{n}$ then

$$
\operatorname{ad}(\Phi)=\max \left(\operatorname{ad}\left(\Phi^{\prime}\right), \operatorname{ad}\left(\Gamma_{1}\right), \ldots, \operatorname{ad}\left(\Gamma_{n}\right)\right)
$$

where $\Phi^{\prime}$ is obtained from $\Phi$ by substituting new atomic propositions $A_{1}, \ldots, A_{n}$ for $\Gamma_{1}, \ldots, \Gamma_{n}$.

- If $\Phi$ contains no closed top-level fixpoint-subformulas then $\operatorname{ad}(\Phi)$ is defined as follows.

$-\operatorname{ad}(A)=\operatorname{ad}(X)=0$, for any atomic proposition $A$ and variable $X$.

$-\operatorname{ad}\left(\Phi_{1} \wedge \Phi_{2}\right)=\operatorname{ad}\left(\Phi_{1} \vee \Phi_{2}\right)=\max \left(\operatorname{ad}\left(\Phi_{1}\right), \operatorname{ad}\left(\Phi_{2}\right)\right)$.

$-\operatorname{ad}([a] \Phi)=\operatorname{ad}(\langle a\rangle \Phi)=\operatorname{ad}(\Phi)$, for any action $a$. 
- Let $\sigma \in\{\mu, \nu\}$, and let $\bar{\sigma}$ be the dual of $\sigma$. Then

$$
\operatorname{ad}(\sigma X . \Phi)=\max \left(1, \operatorname{ad}(\Phi), 1+\operatorname{ad}\left(\bar{\sigma} X_{1}, \Phi_{1}\right), \ldots, 1+\operatorname{ad}\left(\bar{\sigma} X_{n} . \Phi_{n}\right)\right)
$$

where $\bar{\sigma} X_{1} . \Phi_{1}, \ldots, \bar{\sigma} X_{n} \cdot \Phi_{n}$ are the top-level $\bar{\sigma}-$ subformalas of $\Phi$.

Example 2.5 For $\Phi=\nu X_{1}, \mu X_{2} \cdot\left(X_{1} \vee X_{2} \vee \nu Y_{1}, \mu Y_{2} . \nu Y_{3} \cdot\left(Y_{1} \wedge Y_{2} \wedge Y_{3}\right)\right)$ we obtain $\operatorname{ad}(\Phi)=3$.

\section{Equational Systems}

In order to facilitate the saving and reuse of intermediate results, our model-checking algorithm works on equational representations of mu-calculus formulas. This section presents the syntax and semantics of the equational systems and introduces the notions of closed subsystems and alternation depth.

\subsection{Syntax of Equational Systems}

The systems of mutually recursive equations that we use to represent formulas are lists of the following form ${ }^{1}:\left\langle X_{1} \Phi_{1} \Phi_{1}, \ldots, X_{n} \Phi_{n} \Phi_{n}\right\rangle$ where $\Phi_{i} \in\{\rightarrow, \leftarrow\}$. The $X_{i}$ 's are distinct variables, and the equation $X_{i} \rightarrow \Phi_{i}$ represents a grealest fixpoint, while $X_{i} \leftarrow \Phi_{i}$ represents a least fixpoint. Following [AC, CS1] we restrict our attention to mu-calculus formulas $\Phi_{i}$ that are negation-free and simple, which guarantees that every non-atomic right-hand-side formula has a left-hand-side variable associated with it. This facilitates the saving and reuse of intermediate results. Any equation set $E$ may be transformed in linear time into a simple equational system $E^{\prime}$ with at most linear blow-up in size. Therefore, the model-checking algorithm presented in this paper has the same complexity for the full logic as for the simple sublogic. In what follows we refer to $X_{i} \rightarrow \Phi_{i}$ as a maz equation with max variable $X_{i}$ and to $X_{j} \leftarrow \Phi_{j}$ as a min equation with min variable $X_{j}$, and we associate with each left-hand-side variable a parity that is either max or min depending on the form of the equation. An equational system $E$ is closed if all variables in a right-hand side of some equation also appear as left-hand sides in $E$. It should be pointed out that the order of equations is important in an equational system, owing to the presence of mutually recursive greatest and least fixpoint formulas.

Example 3.1 The following equational system $E$ represents the formula given in Example 2.5. It can be obtained by means of the translation that will be given in Section 3.2 .

$$
\left\langle X_{1} \rightarrow X_{2}, X_{2} \leftarrow X_{1} \vee X_{3}, X_{3} \leftarrow X_{2} \vee X_{4}, X_{4} \rightarrow X_{5}, X_{5} \leftarrow X_{6}, X_{6} \rightarrow X_{4} \wedge X_{7}, X_{7} \rightarrow X_{5} \wedge X_{6}\right)
$$

\subsection{Semantics of Equational Systems}

The semantics for equational systems uses a translation from systems of equations to tuples of closed mu-calculus formulas, one for each equation. An equational system may then be interpreted as a tuple of subsets of states which arises by pointwise application of the semantic function for formulas to the component formulas.

This translation consists of the composition of two functions, $B$ and $F$ (for "backwards" and "forwards"), which repeatedly eliminate occurrences of free variables. Let $E=\left\langle X_{1} \oplus \Phi_{1}, \ldots, X_{n} \oplus \Phi_{n}\right\rangle$ be a closed, simple equational system, and let $\bar{\Phi}=\left\langle\Phi_{1}, \ldots, \Phi_{n}\right\rangle$ consist of the right-hand sides of $E$. Also let $\pi_{1}, \ldots, \pi_{n}$ be the obvious projection functions. Given $\bar{\Phi}, B$ produces a new tuple $\bar{\Gamma}$ of formulas by setting $\bar{\Gamma}$ to $\bar{\Phi}$ and processing each component in $\bar{\Gamma}$ as follows, beginning with $\pi_{n}(\bar{\Gamma})$ and working backwards.

- Replace $\pi_{i}(\bar{\Gamma})$ by $\mu X_{i} \cdot \pi_{i}(\bar{\Gamma})$ (if $X_{i}$ is a min-variable) or $\nu X_{i} \cdot \pi_{i}(\bar{\Gamma})$ (if $X_{i}$ is a max-variable).

\footnotetext{
'This form is similar to the one used by Larsen in [La].
} 


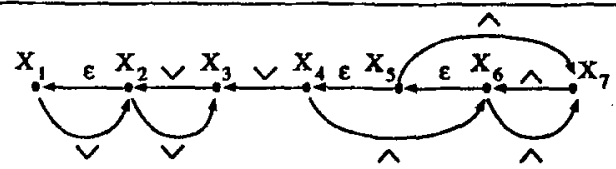

Figure 2: The Dependency Graph for Equational System $E$ in Example 3.1.

- Substitute $\pi_{i}(\bar{\Gamma})$ for each free occurrence of $X_{i}$ in $\pi_{1}(\bar{\Gamma}), \ldots, \pi_{i-1}(\bar{\Gamma})$.

Note that only $X_{1}, \ldots, X_{i-1}$ can appear free in $\pi_{i}(B(\bar{\Phi}))$; in particular, $\pi_{1}(B(\bar{\Phi}))$ is closed. Now $F$ eliminates all remaining free variables: Given a tuple $\bar{\Gamma}$ of formulas, $F$ produces a new tuple $\bar{\Delta}$ by processing each formula in $\bar{\Gamma}$ in the order of the indices as follows: substitute $\pi_{i}(\bar{\Delta})$ for each free occurrence of $X_{i}$ in $\pi_{i+1}(\bar{\Delta}), \ldots, \pi_{n}(\bar{\Delta})$. The semantics of $E$ can now be given as follows.

Definition 3.2 (Semantics of Equational Systems) Let $E$ be a closed, simple system of $n$ equations, and let $\bar{\Phi}$ be the tuple of right-hand sides of $E$. Also let $\left\langle\Delta_{1}, \ldots, \Delta_{n}\right\rangle=F \circ B(\bar{\Phi})$. Then $[E]=\left\langle\llbracket \Delta_{1} \rrbracket, \ldots, \llbracket \Delta_{n} \rrbracket\right\rangle$.

The connection between equational systems and the mu-calculus can be made explicit by providing translations back and forth. trans ${ }^{a}$, translating equational systems into formulas, is straightforward in terms of $F$ and $B$ : trans ${ }^{a}(E)=\dot{\pi}_{1}(F \circ B(\Phi))$, where $\bar{\Phi}$ consists of the right-hand sides of $E$. Given a mu-calculus formula $\Phi$ in PNF, the function trans builds an equational system by recursing through $\Phi$, adding a new equation at the end of the last of the already generated equations for each subformula of $\Phi$. The parity of a new added left-hand-side variable is determined by the most recently encountered fixpoint operator. As an example, consider the formula and the equational system given in Example 2.5 and 3.1, respectively. Here, the application of trans to $\Phi$ yields $E$.

Obviously, trans works in linear time as every subformula of $\Phi$ is investigated exactly once. Moreover, the number of equations in the resulting simple equational system $E_{W}$ is less than or equal to the size of the formula $\Phi$, because every subformula of $\Phi$ is transformed into at most one equation. A detailed account of these translations can be found in [CDS].

Instead of solving the model-checking problem directly for a given formula $\$$ we solve it on the equational system $E_{\boldsymbol{E}}$ that is gained by the translation given above. The following theorem establishes the correctness of this approach.

Theorem 3.3 Let $\Phi$ be a closed $P N F$ formula and $E_{\uparrow}=\operatorname{trans}(\Phi)$. Then, $[\Phi]=\pi_{1}\left(\left[E_{\downarrow}\right]\right)$.

\subsection{Graph Representation of Equational Systems}

In this section we introduce a graph representation of equational systems that will be used to determine the closed subsystems of equational systems and to define the notion of alternation depth. Let $E$ be an equational system. Then its dependency graph $G_{E}$ is an edge-labeled graph with one node for each left-hand-side variable in $E$ and edges defined as follows, where $i \neq j$.

- $X_{i} \stackrel{b}{\rightarrow} X_{j}$ if for some $\Phi$ either $X_{j} \diamond X_{i} l \Phi$ or $X_{j} \bowtie \Phi l X_{i}$ is an equation in $E$ for $l \in\{\vee, \wedge\}$.

- $X_{i} \stackrel{b}{\rightarrow} X_{j}$ if $X_{j} \diamond l X_{i}$ is in $E$ for $l \in\{\langle a\rangle,[a]\}$.

- $X_{i} \stackrel{\bullet}{\rightarrow} X_{j}$ if $X_{j} \diamond X_{i}$ is in $E$.

Intuitively, there is an edge from $X_{i}$ to $X_{j}$ if the meaning of $X_{i}$ directly influences the meaning of $X_{j}$. In what follows, we write $X_{i} \rightarrow X_{j}$ if there is an edge in $G_{E}$ from $X_{i}$ to $X_{j}$ and $X_{i} \dot{\rightarrow}^{*} X_{j}$ if there is a path from $X_{i}$ to $X_{j}$ in $G_{E}$. As an example, the graph for the equational system in Example 3.1 appears in Figure 2. 


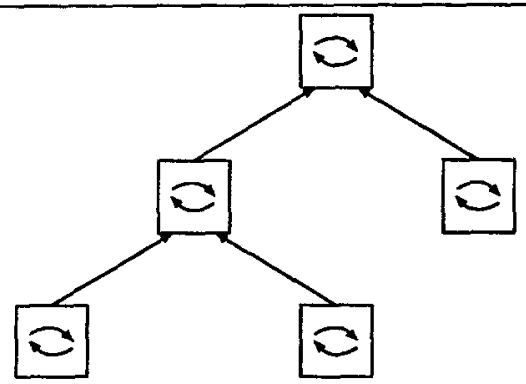

Figure 3: The Dependencies between and within the Closed Subsystems.

Theorem 3.4 Let $E=\left\langle X_{1} \otimes \Phi_{1}, \ldots, X_{n} \otimes \Phi_{n}\right\rangle$ be an equational system. Its dependency graph $G_{E}$ can be constructed in $O(|E|)$ time, and it contains $n$ vertices and no more than $2 n$ edges.

Let $C$ be a sublist of $E$. Then we refer to the subgraph of $G_{E}$ induced by $C$ as $G_{C}$. Also, we write $X_{i} \dot{\rightarrow} C, k X_{j}$ if $X_{i} \rightarrow X_{j}$ is an edge in $G_{C}$ with $i \geq k$ and $j \geq k$. These notions are used in Section 3.5.

\subsection{Closed Subsystems of Equational Systems}

In analogy with the notion of closed subformulas, we develop the notion of closed subsystems of equational systems; these turn out to be essential in order for us to achieve the desired complexity for our model-checking algorithm.

From the definition of the dependency graph $G_{E}$, if two variables $X_{i}$ and $X_{j}$ are such that $X_{i} \rightarrow^{*} X_{j}$ and $X_{j} \dot{\rightarrow}^{*} X_{i}$, it follows that the semantics of $X_{i}$ affects that of $X_{j}$, and vice versa. When this is the case we say that $X_{i}$ and $X_{j}$ are mutually dependent, since any change to the semantics of one may induce a change in the other. On the other hand, if $X_{i} \rightarrow^{*} X_{j}$ but $X_{j} \neq^{*} X_{i}$, then changes to $X_{i}$ affect $X_{j}$, but not vice versa. In this case we say that there is a hierarchical dependency from $X_{i}$ to $X_{j}$, since once the semantics of $X_{i}$ is computed future changes to $X_{j}$ cannot affect it.

In graph-theoretic terms, when $X_{i} \dot{\rightarrow}^{*} X_{j}$ and $X_{j} \dot{\rightarrow}^{*} X_{i}$, then $X_{i}$ and $X_{j}$ belong to the same strongly connected component of $G_{E}{ }^{2}$ Within a strongly connected component each pair of variables is mutually dependent, while there can exist at most a hierarchical dependence between two variables in distinct strongly connected components. This suggests the following strategy for computing 【E]:

1. Build the condensation graph, $G_{C}$, of $G_{E}$. (Recall that the condensation graph of $G$ is a graph having the strongly connected components $G_{i}$ of $G$ as its vertices, with an edge $G_{i} \rightarrow G_{j}$ defined if $G_{i}$ and $G_{j}$ are distinct and there are nodes $V_{i} \in G_{i}, V_{j} \in G_{j}$, such that $V_{i} \rightarrow V_{j}$ is an edge in $G$.) Note that $G_{C}$ is acyclic.

2. Topologically sort $G_{C}$ into $G_{m}, \ldots, G_{1}$. (Here $G_{m}$ is a "source" node in $G_{C}$; we have elected to number it $m$ so that, in general, higher-numbered variables belong to higher-numbered components.) Notice that if there is an edge from $G_{i}$ to $G_{j}$ then $i>j$.

3. For each $G_{i}$, generate a closed subsystem $C_{i}$ containing the equations from $E$ whose left-hand sides are in $G_{i}$. These equations are modified by replacing each occurrence of $X_{i}$ that is not a left-hand side in $G_{i}$ by a new atomic proposition $A_{j}$; this ensures that $C_{i}$ is closed. Note that if $X_{j}$ is in component $G_{k}$ then $k<i$.

\footnotetext{
${ }^{2}$ Recall that a strongly connected component of a graph is a maximal subet $\mathcal{V}$ of vertices having the property that $V_{i} \rightarrow^{*} V_{j}$ and $V_{j} \rightarrow V_{i}$ for any $V_{i}, V_{j} \in V$.
} 
4. Beginning with $C_{m}$, process each $C_{i}$ in turn.

As an example, consider $E$ in Example 3.1 with its dependency graph $G_{E}$ shown in Figure 2. As there are two strongly connected components of $G_{E}$ we get two closed subsystems:

$$
\begin{gathered}
C_{1}=\left\langle X_{1} \rightarrow X_{2}, X_{2} \leftarrow X_{1} \vee X_{3}, X_{3} \leftarrow X_{2} \vee A_{4}>\right. \\
C_{2}=\left\langle X_{4} \rightarrow X_{5}, X_{5} \leftarrow X_{6}, X_{6} \rightarrow X_{4} \wedge X_{7}, X_{7} \rightarrow X_{5} \wedge X_{6}>\right.
\end{gathered}
$$

Note that each $C_{l}$ is closed and that each left-hand-side variable $X_{i}$ of $E$ appears as a lefthand side in exactly one of the $C_{l}$. Also notice that the construction ensures that if a new atomic proposition $A_{i}$ appears in a right-hand side in $C_{j}$, then $X_{i}$ must appear as a left-hand side in some $C_{l}$ with $l>j$. Consequently, we may define the semantics of $A_{i}$ as follows. Let $C_{l}$ be the closed subsystem containing $X_{i}$ as a left-hand sje, and let $k$ be the index of $X_{i}$ in $C_{l}$. Then $\llbracket A_{i} \rrbracket=\pi_{k}\left(\left[C_{l} \rrbracket\right)\right.$. The following theorem shows that this transformation of $E$ into $C_{1} \ldots C_{m}$ is, in a certain sense, semantics-preserving.

Theorem 3.5 The closed subsystems $C_{1}, \ldots, C_{m}$ of an equational system $E$ can be determined in $O(|E|)$ time. Furthermore, if $X_{i}$ is the $k^{\text {th }}$ left-hand side in $C_{l}$, then $\pi_{i}([E])=\pi_{k}\left(\left[C_{l} \mid\right)\right.$.

In our example we have $\llbracket E]=\left\langle x_{1}\left[C_{1}\right\rfloor, \pi_{2} \llbracket C_{1} \rrbracket, \pi_{3}\left[C_{1} \rrbracket, \pi_{1}\left[C_{2}\right], \pi_{2}\left[C_{2}\right], \pi_{3}\left[C_{2}\right], \pi_{4}\left[C_{2}\right\rfloor\right\rangle\right.$.

\subsection{Alternation Depth of Equational Systems}

We close this section by defining the notion of allernation depth of an equational system. It will turn out that this notion is consistent with the one given for formulas (cf. Theorem 3.8), and therefore we may use the same notation.

To define the alternation depth we first introduce the notion of nesting depth of equations that reflects the length of the chain of mutually depending $\min$ and max equations within a closed subsystem.

\section{Definition 3.6 (Nesting Depth of Equations)}

Let $E=\left\langle X_{1} \otimes \Phi_{1}, \ldots, X_{n} \oplus \Phi_{n}\right\rangle$ be an equational system with its closed subsystems $C_{1}, \ldots, C_{m}$. Furthermore, assume $\sigma \in\{\max , \min \}$ and $\bar{\sigma}$ to be the dual parity. Then the nesting depth of the equation with left-hand side $X_{i}$ having parity $\sigma$ and belonging to $C_{l}$ is given by:

$$
\begin{aligned}
\operatorname{nd}\left(X_{i}, C_{l}\right)=\max (1, & \max \left\{n d\left(X_{j}, C_{l}\right) \mid X_{j} \dot{\rightarrow} C_{l, i}{ }^{*} X_{i} \text { and } X_{j} \text { has parity } \sigma\right\}, \\
& \left.\max \left\{1+\operatorname{nd}\left(X_{j}, C_{l}\right) \mid X_{j} \rightarrow c_{l, i}{ }^{*} X_{i} \text { and } X_{j} \text { has parity } \bar{\sigma}\right\}\right)
\end{aligned}
$$

The nesting depth of the closed subsystem $C_{l}$ is defined as $n d\left(C_{l}\right)=\max \left\{\operatorname{ndd}\left(X_{i}, C_{l}\right) \mid X_{i} \bowtie \Phi_{i} \in C_{l}\right\}$.

The alternation depth of an equational system is now defined as the maximal nesting depth of its closed subsystems.

\section{Definition 3.7 (Alternation Depth of Equational Syatems)}

Let $E=\left\langle X_{1} \otimes \Phi_{1}, \ldots, X_{n} \otimes \Phi_{n}\right\rangle$ be an equational system with closed subsystems $C_{1}, \ldots, C_{m}$. Then the alternation depth of $E$ is given by $\operatorname{ad}(E)=\max \left\{n d\left(C_{l}\right) \mid 1 \leq l \leq m\right\}$.

Example: As shown already, the equational system $E$ presented in Example 3.1 has two closed subsystems, and we have: $\operatorname{nd}\left(X_{3}, C_{1}\right)=\operatorname{nd}\left(X_{2}, C_{1}\right)=1$ and $n d\left(X_{1}, C_{1}\right)=2$, thus $\operatorname{nd}\left(C_{1}\right)=2$ and $n d\left(X_{7}, C_{2}\right)=n d\left(X_{6}, C_{2}\right)=1, n d\left(X_{6}, C_{2}\right)=2$ and $\operatorname{nd}\left(X_{1}, C_{2}\right)=3$, thus $n d\left(C_{2}\right)=3$. Therefore $\operatorname{ad}(E)=3$.

We say that an equational system $E$ is alternation-free if $a d(E)=1$. The consistency of the notions of alternation depth for formulas and equational systems is a consequence of the following theorem. 
Theorem 3.8 Let $\Phi$ be a closed PNF formula with ad $(\Phi) \geq 1$ and $E_{\Phi}=\operatorname{trans}(\Phi)$ be the corresponding equational system. Then $\operatorname{ad}(\Phi)=\operatorname{ad}\left(E_{\Phi}\right)$.

The left-hand-side variables of a closed subsystem of an equational system can be partitioned into nesting levels, which are used to guide the fixpoint computation.

Definition 3.9 (Nesting Levels) Let $E=\left(X_{1} \otimes \Phi_{1}, \ldots, X_{n} \otimes \Phi_{n}\right)$ be an equational system with closed subsystems $C_{1}, \ldots, C_{\mathrm{m}}$. Then the set of variables belonging to a closed subsystem $C_{l}$ is partitioned into nesting levels by $E_{l, i}=\left\{X_{j} \mid n d\left(X_{j}, C_{l}\right)=i\right\}$ for $1 \leq i \leq n d\left(C_{l}\right)$.

Given a nesting level $E_{l, i}$ we call the nesting level $E_{l, j}$ lower if $j<i$ and higher if $j>i$. Each nesting level consists of at most two blocks of equations, where a block consists entirely of min or of max equations.

Theorem 3.10 Given an equational system E:

1. Alternation-freedom can be established in $O(|E|)$ time.

2. The nesting levels can be determined in $O\left(|E|^{2}\right)$ time.

\section{The Model-Checking Algorithm}

In this section we present a model-checking algorithm that, given an equational system $E$ and a transition system $T=\langle\mathcal{S}, A c h \rightarrow\rangle$, computes $\llbracket E]$. Due to space limitations, we only sketch an outline of the algorithm; the interested reader is referred to [CDS] for a fuller discussion of the details.

As with the algorithms in [AC, CS1, CS2], our algorithm is bit-vector-based. Esch state in $\mathcal{S}$ has a bit vector whose $i^{\text {th }}$ entry indicates whether or not the state belongs to the set associated with $X_{i}$ in the current stage of the analysis. These bit-vectors represent the current approximation $\left(S_{1}, \ldots, S_{n}\right\rangle \in\left(2^{S}\right)^{n}$ to $[E]$ during model checking as follows: $s \in S_{i}$ if and only if $s . X[i]$ is true, for $1 \leq i \leq n$.

Given $E$, the algorithm works by first determining the closed subsystems $C_{1} \ldots C_{m}$ of $E$. It then processes each $C_{l}$ in turn, beginning with $C_{m}$ and ending with $C_{1} ;\left[C_{l}\right]$ is computed and stored in the relevant bit-vector components, and then the atomic predicates whose semantics depend on lefthand sides in $C_{l}$ have their semantics initialized. The algorithm terminates after $C_{1}$ is completed. Given that each $\left[C_{l}\right]$ is computed properly, correctness follows from Theorem 3.5.

At the heart of the algorithm is the computation of $\left[C_{l}\right]$ for a closed subsystem $C_{l}$. This processing proceeds in two phases. During the first phase, bit-vectors are initialized such that components corresponding to max variables are set to true and components corresponding to min variables are set to false. In the second phase, the nesting levels of $C_{l}$ are repeatedly analyzed,

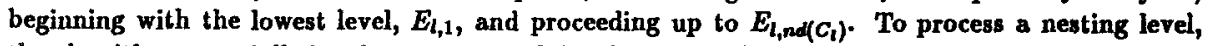
the algorithm essentially invokes a variant of the alternation-free model-checking algorithm given in [CS2]. Bit-vector annotations are changed until appropriate fixed points are reached; in addition, if changing a bit-vector component in one variable also causes a change in the semantics of a variable in a lower nesting level, then the lower nesting levels that are affected must be re-initialized and recomputed. The processing of a nesting level is finished when consistency is reached with all lower levels. Then, the next higher level is begun.

In this computation of $\left[C_{l} \rrbracket\right.$, one may identify two flows of information.

- The flow of assumptions: Our algorithm may be seen as "assumption based": during the computation of a fixpoint for equations in a nesting level, the variables in higher nesting levels are treated essentially as propositional constants in that their meaning is fixed. Thus, the assumption flow proceeds from $E_{l, n d\left(C_{l}\right)}$ down to $E_{l, 1}$. 


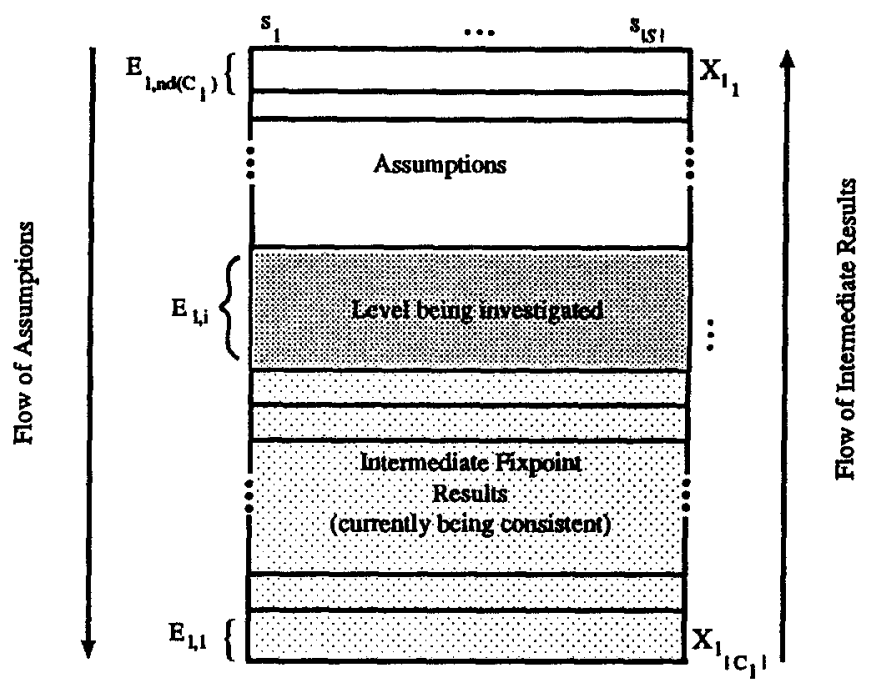

Figure 4: The Flows of Information in a Closed Subsystem $C_{l}$.

- The propagation of intermediate results: Fixpoints are computed from lower to higher nesting levels. Therefore, the computation flow proceeds in the direction opposite to that of the flow of assumptions, as intermediate results computed in one level may affect the results for higher levels.

In this view, the need for recomputing values in lower nesting levels when a higher nesting level changes becomes apparent: the computation of the lower level was based on a wrong assumption.

The two flows of information are illustrated in Figure 4, where the box represents the current approximation of the semantics of $C_{l}$ with the bit-vectors corresponding to columns through the levels.

Three observations are exploited in order to achieve the complexity stated in the next section.

1. The partitioning of the equational system $E$ into closed subsystems $C_{1}, \ldots, C_{m}$ ensures that once $\llbracket C_{l} \rrbracket$ is computed, it cannot be affected by the analysis of subsequent closed subsystems.

2. Within a given closed subsystem $C_{t}$ the nesting levels are treated exactly as in the (linear-time [CS2]) alternation-free case each time their fixpoint is computed.

3. Computing consistency of the lowest, and most often recomputed, nesting level $E_{l, 1}$ is less expensive than for the higher levels as $E_{l, 1}$ does not give rise to resetting and recomputation of lower levels and also need not account for the new values that resetting and recomputing lower levels can give rise to.

The full structure of the model-checking algorithm is given in [CDS]; Section 6 contains an example illustrating our technique.

\section{Correctness and Complexity}

The correctness of the algorithm rests on the observation that our algorithm computes $\left[C_{l}\right]$ component-wise according to the semantic definition of formulas by representing the environment in the bit vectors. Together with Theorem 3.5 this enables us to prove the following theorem (cf. [CDS]). 


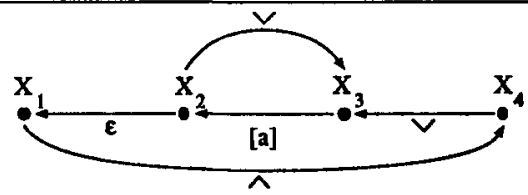

Figure 5: The Dependency Graph.

Theorem 5.1 (Correctness) Let $\mathcal{T}=\langle\mathcal{S}$, Ach $\rightarrow\rangle$ be a labeled finite-state transition system and $E=\left\langle X_{1} \bowtie \Phi_{1}, \ldots, X_{n} \bowtie \Phi_{n}\right\rangle$ be a closed, simple equational aystem. Then the model-checking algorithm terminates with a bit-vector annotation that represents [E].

The following theorem states our complexity result, where $|\mathcal{T}|=|\mathcal{S}|+|\rightarrow|$ and $|E|$ is the number of equations in $E$. A complete proof is given in [CDS].

Theorem 5.2 (Complexity) Let $E=\left\langle X_{1} \diamond \Phi_{1}, \ldots, X_{n} \diamond \Phi_{n}\right\rangle$ be a simple, closed equational system with $\operatorname{ad}(E) \geq 1$, and $\mathcal{T}=\langle\mathcal{S}$, Ach $\rightarrow\rangle$ be a finite-state transition system. Then the worstcase time complexity of the model-checking algorithm is

$$
O\left(|T| *|E| *\left(|\mathcal{S}| * \frac{|E|}{\operatorname{ad}(E)}\right)^{\operatorname{ad}(E)-1}\right)
$$

\section{An Example}

In this section we illustrate our algorithm with an example taken from [SW]. Consider the formula $\Phi=\nu Z, \mu Y \cdot[a]((A \wedge Z) \vee Y)$ having alternation depth 2. The semantics of with respect to a transition system $T$ is the set of states for which $A$ holds infinitely often on all a-paths. Its corresponding equational system

$$
E=\left\langle X_{1} \rightarrow X_{2}, X_{2} \leftarrow[a] X_{3}, X_{3} \leftarrow X_{4} \vee X_{2}, X_{4} \leftarrow A \wedge X_{1}\right\rangle
$$

only has the trivial closed subsystem consisting of two nesting levels: $E_{1}$ holding the last three equations, and $E_{2}$ holding the first equation. The dependency graph is shown in Figure 5.

The transition system $\mathcal{T}$ we want to investigate is the triple $\langle\mathcal{S}$, Act $\rightarrow\rangle$, where $\mathcal{S}=\{s, t, u, v\}$, $A c t=\{a\}$ and the transition relation has six elements: $\stackrel{a}{\rightarrow} s, s \stackrel{a}{\rightarrow} t, t \stackrel{a}{\rightarrow} u, u \stackrel{a}{\rightarrow} s, u \stackrel{a}{\rightarrow} v$ and $v \stackrel{a}{\rightarrow} v$.

The valuation is given by $\mathcal{V}(A)=\{t, u, v\}$; so states $t, u$ and $v$ satisfy $A$, but $s$ does not. Besides the bit-vectors s.X[1..4], t.X[1..4], u.X $[1 . .4]$ and $v . X[1 . .4]$ we need some auxiliary data structures for investigating the levels (cf. [CS2]): the counters s.C[1..4], t.C[1..4], u.C[1..4] and v.C[1..4], where $x . C[i]$ maintains a count of the number of components $y . X[j]$ that may change until $x . X[i]$ must change; and the array of worklists $M[1 . .4]$, where $M[i]$ holds the states the changes to whose $i^{\text {th }}$ bit-vector components have yet to be propagated. The states also contain fields recording whether they satisfy the atomic formula $A$; so $s . A=f$, while $t . A=u . A=v . A=t$. Note that $X_{1}$ is a $\max$ variable initialized with true for all states and $X_{2}, X_{3}$ and $X_{4}$ are min variables accordingly initialized with false. In what follows wë highlight the changes made to the data structure step by step. Note in particular the change of intermediate results in $E_{1}$ because of changing assumptions in $E_{2}$.

- Computing a fixpoint over the lowest level $E_{1}$ (containing $X_{2}$ to $X_{4}$ ) starts with the following

\begin{tabular}{|c|c|c|c|c|c|c|c|c|c|c|}
\hline & 8 & $t$ & $u$ & 0 & & s & $t$ & $\mathbf{u}$ & $\boldsymbol{v}$ & \\
\hline $\begin{array}{l}X_{1} \\
X_{2}\end{array}$ & ut & $\boldsymbol{t} t$ & $f$ & $t$ & $C_{1}$ & 1 & 1 & 2 & 1 & $M[\theta, \theta, \theta,\{t, u, v\}]$ \\
\hline$X_{3}$ & ff & ff & f & 0 & $C_{3}$ & 1 & 1 & 1 & 1 & \\
\hline$X_{1}$ & ff & $t t$ & tt & $t$ & $C_{4}$ & 1 & 0 & 0 & 0 & \\
\hline
\end{tabular}
initialization of the bit vectors, counters and worklists. 
The influence of the states in the worklist is determined. First, $t, u, v$ are successively deleted from $M[4]$ and $X_{4} \stackrel{\vee}{\rightarrow} X_{3}$ is processed. Second, $t, u, v$ are successively deleted from $M[3]$ and $X_{3} \stackrel{[a]}{\rightarrow} X_{2}$ is processed. This provides the following intermediate results:

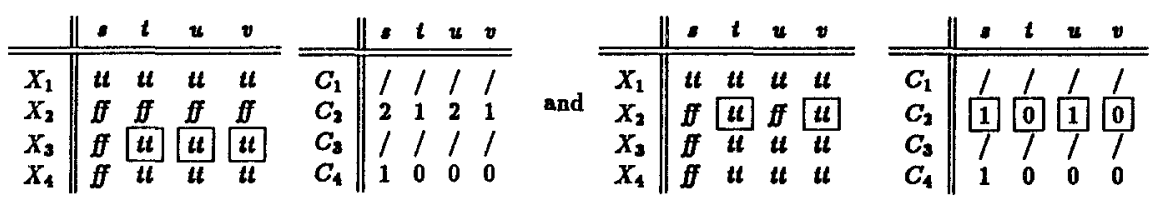

$$
M[\theta, \theta,\{t, u, v\}, \theta]
$$

$M[\theta,\{t, v\}, \theta, \theta]$

Now $t$ and $v$ are successively deleted from $M[2]$ and $X_{2} \stackrel{v}{\rightarrow} X_{3}$ is processed. As $t . X[3]$ and $v . X[3]$ are already true the bit-vectors remain unchanged and the worklists for $E_{1}$ are empty.

- On the next nesting level $E_{2}$ the fixpoint computation detects an inconsistency for $s$ and an inconsistency for $u$ as s.X[1] $=u . X[1]=t$ but s. $X[2]=u . X[2]=f$ and $X_{2} \stackrel{e}{\rightarrow} X_{1}$. Thus s.X[1] and $u . X[2]$ are set to false and $E_{1}$ has to be reset and recomputed accordingly.

- The recomputation of $E_{1}$ taking the new assumptions into account starts with the initialization shown on the left and computes the fixpoint shown on the right.

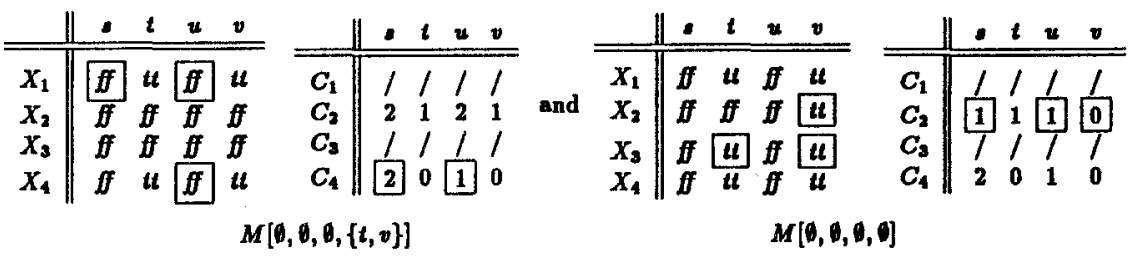

- Again computing the fixpoint over $E_{2}$ an inconsistency is detected as $t \cdot X[1]=t t$ but $t \cdot X[2]=$ $f f$ and $X_{2} \stackrel{e}{\rightarrow} X_{1}$. Thus $t . X[1]$ is set to false and $E_{1}$ is reset and recomputed, providing the following results for initialization (left) and fixpoint computation (right):

\begin{tabular}{l||llll} 
& $:$ & $t$ & $u$ & $v$ \\
\hline \hline$X_{1}$ & $f$ & {$[f]$} & ff & $u$ \\
$X_{2}$ & $f f$ & $f f$ & ff & $f f$ \\
$X_{3}$ & $f$ & ff & ff & ff \\
$X_{4}$ & $f f$ & f & ff & $u$
\end{tabular}

$M[\theta, \theta, \theta,\{v\}]$$$
\text { ) }
$$

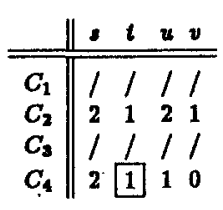

Finally, $E_{2}$ is shown to be consistent, the algorithm terminates, and we obtain $[\Phi]=\{v\}$, as $v . X[1]=t$ and the first bit-vector component of all other states is false. This reflects one's intuition about the formula, because $v$ is the only state for which $A$ is infinitely often satisfied along all a-paths.

\section{Conclusions and Future Work}

In this paper, we have presented an algorithm for model checking that handles the full modal mucalculus including alternating fixed points. The algorithm extends the one given in [CS2] for an alternation-free logic. Central is the new complexity result:

$$
O\left(|T| *|E| *\left(|S| * \frac{|E|}{\operatorname{ad}(E)}\right)^{\operatorname{ad}(E)-1}\right)
$$


which improves even on our conjecture ([CS2]):

- Instead of being exponential in the full size of the transition system it is only exponential in the number of its states. This saves a quadratic blow-up in the worst case.

- Instead of being exponential in the full size of the formula, it is only exponential in $\frac{|E|}{\operatorname{ad}(E)}$, which is important for formulas with high alternation depth.

In [A] Andersen sketches an $O\left(|S| *|T|^{\text {ad( }(\$)-1} *|\Phi|^{\text {ad( }(\$)}\right)$ algorithm for the full mu-calculus, which improves on Emerson and Lei's result, $O\left((|T| *|E|)^{-\alpha(E)+1}\right)$. Andersen's algorithm differs from ours in that it is tailored to the mu-calculus structure rather than systems of equations, where properties can be expressed much more concisely. In the worst case, his formalizations are exponentially larger than ours, because equational systems allow to compactly represent common subexpressions. This generality, however, requires a much more involved algorithm. Nevertheless, we were able to prove a stronger complexity result, even with respect to the more compact representations. Our algorithm will be implemented as an extension of the Concurrency Workbench [CPS1, CPS2].

\section{References}

[A] Andersen, H. "Model Checking and Boolean Graphs." Proc. of ESOP '92, LNCS 582, 1992.

[AC] Arnold, A., and P. Crubille. "A Linear Algorithm To Solve Fixed-Point Equations on Transition Systems." Information Processing Letters, v. 29, 30 September 1988, pp. 57-66.

[CES] Clarke, E.M., E.A. Emerson and A.P. Sistla. "Automatic Verification of Finite State Concurrent Systems Using Temporal Logic Specifications." ACM Transactions on Programming Languages and Systems, v. 8, n. 2, 1986, pp. 244-263.

[C] Cleaveland, R. "Tableau-Based Model Checking in the Propositional Mu-Calculus." Acta Informatica, v. 27, 1990, pp. 725-747.

[CDS] Cleaveland, R., M. Klein and B. Steffen. "Faster Model Checking for the Modal MuCalculus." In Technical Report RWTH Aachen Nr. 91-29, Fachgruppe der Informatik, 1991.

[CPS1] Cleaveland, R., J. Parrow and B. Steffen. "The Concurrency Workbench." In Proceedings CAV'89, LNCS 407, 1989.

[CPS2] Cleaveland, R., J. Parrow and B. Steffen. "A Semantics-based Verification Tool for FiniteState Systems", In Proceedings of the Ninth International Symposium on Protocol Specifcation, Testing, and Verification. North-Holland, 1989.

[CS1] Cleaveland, R. and B. Steffen. "Computing Behavioural Relations, Logically." In Proceedings ICALP '91, LNCS 510, 1991.

[CS2] Cleaveland, R. and B. Steffen. "A Linear-Time Model Checking Algorithn for the Alternation-Free Modal Mu-Calculus." In Proceedings CAV '91, LNCS 575, 1991.

[EL] Emerson, E.A. and C.-L. Lei. "Efficient Model Checking in Fragments of the Propositional Mu-Calculus." In Proceedings of LICS, 1986, pp. 267-278.

[Fe] Fernandez, J.-C. Aldébaran: Une Système de Vérification par Réduction de Processus Communicants. Ph.D. Thesis, Université de Grenoble, 1988.

[K] Kleene, S. C. "Introduction to Metamathematics", North Holland, 1952.

[Ko] Kozen, D. "Results on the Propositional $\mu$-Calculus." TCS, v. 27, 1983, pp. 333-354. 
[La] Larsen, K.G. "Proof Systems for Hennessy-Milner Logic with Recursion." In Proceedings of CAAP, 1988.

[MSGS] Malhotra, J., Smolka, S.A., Giacalone, A. and Shapiro, R. "Winston: A Tool for Hierarchical Design and Simulation of Concurrent Systems." In Proceedings of the Workshop on Specification and Verification of Concurrent Systems, Univ. of Stirling, Scotland, 1988.

[RRSV] Richier, J., Rodriguez, C., Sifakis, J. and Voiron, J.. "Verification in XESAR of the Sliding Window Protocol." In Proceedings of the Seventh IFIP Symposium on Protocol Specification, Testing, and Verification, 1987, North-Holland.

[RdS] Roy, V. and R. de Simone. “Auto/Autograph.” In Proceedings, CAV'90, LNCS 531, 1990.

[Ste] Steffen, B.U. "Characteristic Formulae." In Proceedings ICALP, LNCS 372, 1989.

[SI] Steffen, B.U., and A. Ingblfsdóttir. "Characteristic Formulae for CCS with Divergence." To appear in Information and Computation.

[SW] Stirling, C. and D. Walker. "Local Model Checking in the Modal Mu-Calculus." In Proceedings of TAPSOFT' '89, LNCS 351, 1989.

[T] Tarski; A. "A Lattice-Theoretical Fixpoint Theorem and its Applications." Pacific Journal of Mathematics, v. 5, 1955. 